\title{
The Effective Potential of the Conformal Factor in Quantum Einstein Gravity
}

\section{Jan-Eric Daum* University of Mainz}

E-mail: daumethep.physik.uni-mainz.de

\section{Martin Reuter}

University of Mainz

E-mail: reuter@thep.physik.uni-mainz.de

\begin{abstract}
The effective potential of the conformal factor in the effective average action approach to Quantum Einstein Gravity is discussed. It is shown, without invoking any truncation or other approximations, that if the theory has has a non-Gaussian ultraviolet fixed point and is asymptotically safe the potential has a characteristic behavior near the origin. This behavior might be observable in numerical simulations.
\end{abstract}

Workshop on Continuum and Lattice Approaches to Quantum Gravity

17-19 September 2008

University of Sussex, United Kingdom

${ }^{*}$ Speaker. 


\section{Introduction}

One major problem in constructing a fundamental theory of quantum gravity is the complete lack of any experimental data that could be confronted with the corresponding predictions of the theory [1-4]. Therefore, it is particularly important to find out whether some of the a priori different candidate theories are perhaps just different formulations of the same underlying theory or whether they really belong to different "universality classes". All candidates describing the same underlying physics in different formulations must agree on the observations that are within the domain of applicability of the hitherto unknown correct theory of quantum gravity. In this way one can at least narrow down the set of independent possibilities among which the experiment must decide in the end. Guided by the experience with Yang-Mills theory we would expect that in particular the comparison of continuum and lattice approaches should be very instructive and fruitful. On the side of the continuum approaches, recently a lot of efforts went into the exploration of the asymptotic safety scenario [5-28] in the formulation based upon the gravitational average action. It aims at defining a microscopic quantum field theory of gravity in terms of a complete, i.e., infinitely extended renormalization group (RG) trajectory on the theory space of diffeomorphism invariant functionals of the metric. The limit of an infinite ultraviolet (UV) cutoff is taken by arranging this trajectory to approach a non-Gaussian fixed point (NGFP) at large scales $(k \rightarrow \infty)$. This NGFP of the effective average action is not only instrumental in constructing the quantum field theory by dictating how all generalized couplings must "run" when the UV regulator scale is sent to infinity, it also determines the physical properties of the resulting regulator-free theory at large physical scales, the behavior of propagators at large momenta, for instance. We refer to this quantum field theory of the metric, defined in the continuum by means of the effective average action, as Quantum Einstein Gravity (QEG).

In the following we are going to review how the mere existence of a non-Gaussian fixed point allows us to draw inevitable conclusions about the potential of the conformal factor at small distances. Since this result is not restricted to a specific truncation of the full theory and is related to the very notion of asymptotic safety it is a remarkably robust prediction. In particular it should be possible to confirm it by the corresponding lattice approaches. In this way it might provide an opportunity to transfer the successful cross-fertilization between continuum and lattice approaches in Yang-Mills theory [30-33] to the context of gravity.

\section{The Effective Potential of the Conformal Factor in QEG}

We are interested in the standard effective potential (i.e., the one with vanishing infrared cutoff, $k=0$ ) for the conformal factor of metrics on maximally symmetric spacetimes with the topology of a $d$-dimensional sphere $S^{d}$. The starting point is the exact gravitational effective average action [6] along some RG trajectory, $\Gamma_{k}\left[g_{\mu v}, \bar{g}_{\mu v}\right]$, and the related reduced functional $\bar{\Gamma}_{k}\left[g_{\mu v}\right] \equiv \Gamma_{k}\left[g_{\mu v}, g_{\mu v}\right]$. (The ghost arguments are set to zero and are not indicated explicitly.) The latter functional is assumed to have a representation of the form

$$
\bar{\Gamma}_{k}\left[g_{\mu v}\right]=\sum_{\alpha} \bar{u}_{\alpha}(k) I_{\alpha}\left[g_{\mu v}\right]
$$


where $\left\{I_{\alpha}\left[g_{\mu v}\right]\right\}$ is an infinite set of local and nonlocal "basis" functionals, invariant under diffeomorphisms acting on $g_{\mu v}$, and the $\bar{u}_{\alpha}$ 's are the corresponding running coupling constants. We denote their canonical mass dimensions by $d_{\alpha} \equiv\left[\bar{u}_{\alpha}\right]$. Hence, since $\bar{\Gamma}_{k}$ is dimensionless, $\left[I_{\alpha}\right]=-d_{\alpha}$. The dimensionless running couplings are defined by

$$
u_{\alpha}(k) \equiv k^{-d_{\alpha}} \bar{u}_{\alpha}(k)
$$

so that we may rewrite (2.1) as

$$
\bar{\Gamma}_{k}\left[g_{\mu v}\right]=\sum_{\alpha} u_{\alpha}(k) k^{d_{\alpha}} I_{\alpha}\left[g_{\mu v}\right]
$$

Up to now the metric argument $g_{\mu \nu}$ was completely general. At this point we specialize for metrics on $S^{d}$, with a variable radius $\phi$. We parametrize them as

$$
g_{\mu v}=\phi^{2} \hat{g}_{\mu v}
$$

where $\hat{g}_{\mu v}$ is the metric on the round $S^{d}$ with unit radius, and the conformal factor $\phi$ is position independent. Hence $g_{\mu v}$ is a metric on a round sphere with radius $\phi$. We shall denote the volume of the unit- $S^{d}$ by $\sigma_{d} \equiv \int \mathrm{d}^{d} x \sqrt{\hat{g}}=2 \pi^{(d+1) / 2} / \Gamma((d+1) / 2)$.

We use conventions such that the coordinates $x^{\mu}$ are dimensionless and $\phi$ has the dimension of a length. Hence $\left[g_{\mu v}\right]=-2$, and $\hat{g}_{\mu v}$ is dimensionless, $\left[\hat{g}_{\mu v}\right]=0$.

Without having made any approximation so far, the effective average potential for the conformal factor, $U_{k}(\phi)$, by definition, obtains by inserting the special argument (2.3) into $\bar{\Gamma}_{k}$ :

$$
U_{k}(\phi) \int \mathrm{d}^{d} x \sqrt{\hat{g}} \equiv \bar{\Gamma}_{k}\left[g_{\mu v}=\phi^{2} \hat{g}_{\mu v}\right]
$$

In terms of the expansion (2.2) we have the exact representation

$$
U_{k}(\phi)=\sigma_{d}^{-1} \sum_{\alpha} u_{\alpha}(k) k^{d_{\alpha}} I_{\alpha}\left[\phi^{2} \hat{g}_{\mu v}\right]
$$

or, more explicitly,

$$
U_{k}(\phi)=\sigma_{d}^{-1} \sum_{\alpha} u_{\alpha}(k)(k \phi)^{d_{\alpha}} I_{\alpha}\left[\hat{g}_{\mu v}\right]
$$

To obtain equation (2.6) we exploited that $I_{\alpha}\left[\phi^{2} \hat{g}_{\mu v}\right]=\phi^{d_{\alpha}} I_{\alpha}\left[\hat{g}_{\mu v}\right]$ which holds true since $I_{\alpha}$ has dimension $-d_{\alpha}$. (This relation can be regarded the definition of the canonical mass dimension.)

Eq. (2.6) makes it manifest that if we know a complete RG trajectory $\left\{u_{\alpha}(k), 0 \leq k<\infty\right\}$ we can deduce the exact running potential from it, and in particular its $k \rightarrow 0$ limit, the standard effective potential $U_{\text {eff }}(\phi) \equiv U_{k=0}(\phi)$. Usually we are not in the comfortable situation of knowing trajectories exactly; nevertheless certain important properties of $U_{\text {eff }}(\phi)$ can be deduced on general grounds. For this purpose we shall employ the following decoupling argument which is standard in the average action context [30,32].

The basic observation is that the true, i. e. dimensionful coupling constants $\bar{u}_{\alpha}(k)$ have a significant running with $k$ only as long as the number of field modes integrated out actually depends on $k$. If there are competing physical cutoff scales such as masses or field amplitudes the running 
with $k$ stops once $k$ becomes smaller than the physical cutoff scales. (See Appendix C.3 of [28] for an example.) In the case at hand this situation is realized in a particularly transparent way. The quantum metric is expanded in terms of eigenfunctions of the covariant (tensor) Laplacian $\bar{D}^{2}$ of the metric $\bar{g}_{\mu \nu}$. This metric corresponds to a sphere of radius $\phi$; hence all eigenvalues of the Laplacian are discrete multiples of $1 / \phi^{2}$. As a result, when $k$ has become as small as $k \approx 1 / \phi$, the bulk of eigenvalues is integrated out, and the $\bar{u}_{\alpha}$ 's no longer change much when $k$ is lowered even further. Therefore we can approximate

$$
U_{\text {eff }}(\phi) \equiv U_{k=0}(\phi) \approx U_{k=1 / \phi}(\phi)
$$

In order to make the approximation (2.7) strictly valid we have to be slightly more specific about the precise definition of $U_{k}(\phi)$. The above argument could be spoiled by zero modes of $\bar{D}^{2}$. Therefore we define $\Gamma_{k}$ and $U_{k}$ in terms of a functional integral over the fluctuation modes of the metric with a non-zero eigenvalue of $\bar{D}^{2}$ only. As a result, the actual partition function would obtain by a final integration over the zero modes which is not performed here. The only zero modes relevant in the case at hand are those of the conformal factor. It is therefore important to keep in mind that $U_{\text {eff }}(\phi)$ has the interpretation of an effective potential in which the conformal fluctuations have not yet been integrated out.

Eq. (2.7) has a simple intuitive interpretation in terms of coarse graining: By lowering $k$ below $1 / \phi$ one tries to "average" field configurations over a volume that would be larger than the volume of the whole universe. As this is not possible, the running stops. Note that the $S^{d}$ topology enters here; the finite volume of the sphere is crucial.

With the approximation (2.7) we obtain the following two equivalent representations of $U_{\text {eff }}(\phi)$ in terms of the dimensionless and dimensionful running couplings, respectively:

$$
\begin{aligned}
& U_{\text {eff }}(\phi)=\sigma_{d}^{-1} \sum_{\alpha} u_{\alpha}\left(\phi^{-1}\right) I_{\alpha}\left[\hat{g}_{\mu v}\right] \\
& U_{\text {eff }}(\phi)=\sigma_{d}^{-1} \sum_{\alpha} \bar{u}_{\alpha}\left(\phi^{-1}\right) \phi^{d_{\alpha}} I_{\alpha}\left[\hat{g}_{\mu v}\right]
\end{aligned}
$$

As an application of these representations we consider two special cases.

Let us assume the RG trajectory under consideration has a classical regime between the scales $k_{1}$ and $k_{2}$, meaning that $\bar{u}_{\alpha}(k) \approx \mathrm{const} \equiv \bar{u}_{\alpha}^{\text {class }}$ for $k_{1}<k<k_{2}$. Then (2.9) implies that for $k_{2}^{-1}<$ $\phi<k_{1}^{-1}$, approximately,

$$
U_{\text {eff }}(\phi)=\sigma_{d}^{-1} \sum_{\alpha} \bar{u}_{\alpha}^{\text {class }} I_{\alpha}\left[\hat{g}_{\mu v}\right] \phi^{d_{\alpha}}
$$

As expected, this potential has a nontrivial $\phi$-dependence governed by the classical couplings $\bar{u}_{\alpha}^{\text {class }}$.

Next let us explore the consequences which a non-Gaussian fixed point has for the effective potential. We assume that the dimensionless couplings $u_{\alpha}(k)$ approach fixed point values $u_{\alpha}^{*}$ for $k \rightarrow \infty$. More precisely, we make the approximation $u_{\alpha}(k) \approx u_{\alpha}^{*}$ for $k \gtrsim M$ with $M$ the lower boundary of the asymptotic scaling regime. Then the representation (2.8) tells us that $U_{\text {eff }}(\phi)=$ $\sigma_{d}^{-1} \sum_{\alpha} u_{\alpha}^{*} I_{\alpha}\left[\hat{g}_{\mu v}\right]$ if $\phi \lesssim M^{-1}$. Obviously this potential is completely independent of $\phi$ :

$$
U_{\text {eff }}(\phi)=\text { const for all } \phi \lesssim M^{-1}
$$


In typical applications (see below), $M$ equals the Planck mass $m_{\mathrm{Pl}} \equiv \ell_{\mathrm{Pl}}^{-1}$ so that $U_{\text {eff }}$ is constant for $\phi \lesssim \ell_{\mathrm{Pl}}$.

Eq. (2.11) is our main result. It shows that the existence of an ultraviolet fixed point has a characteristic impact on the effective potential of the conformal factor: Regardless of all details of the RG trajectory, the potential is completely flat for small $\phi$. The interpretation of this result is that for $\phi \lesssim M^{-1}$ the cost of energy (Euclidean action) of a sphere with radius $\phi$ does not depend on $\phi$. Spheres of any radius smaller than $M^{-1}$ are on an equal footing. This is exactly the kind of fractal-like behavior and scale invariance one would expect near the NGFP $[8,10]$.

We emphasize that except for the decoupling relation (2.7) no approximation went into the derivation of this result. It is an exact consequence of the assumed asymptotic safety, the existence of a NGFP governing the short distance behavior. Neither has the theory space been truncated nor have any fields been excluded from the quantization (such as in conformally reduced gravity the transverse tensors, for instance, cf. [21,22]).

On the basis of the above general argument we cannot predict how precisely, or how quickly the effective potential flattens when we approach the origin. However, we expect that its derivative with respect to $\phi^{2}, \partial U_{\text {eff }} / \partial \phi^{2}$, vanishes at $\phi=0$. This has an important physical implication. In general, possible vacuum states of the system (the "universe") can be found from the effective field equation $\delta \Gamma_{k=0} / \delta g_{\mu v}=0$. More specifically, $S^{d}$-type groundstate candidates have a radius $\phi_{0}$ at which $\left(\partial U_{\text {eff }} / \partial \phi^{2}\right)\left(\phi_{0}\right)=0$. (Note that for metrics of the type $g_{\mu v}=\phi^{2} \hat{g}_{\mu v}$ the variation $\delta / \delta g_{\mu v}$ corresponds to a partial derivative with respect to $\phi^{2}$.) Thus we see that thanks to the NGFP a vanishing radius $\phi_{0}=0$ has become a vacuum candidate, the $\phi^{2}$-derivative of $U_{\text {eff }}$ vanishes there. (To qualify as the true vacuum it should be the global minimum.) Hence the universe has an at least metastable stationary state with $\phi=0$, i.e. a state with a vanishing metric expectation value $\left\langle g_{\mu v}\right\rangle=0$. In this state gravity is in a phase of unbroken diffeomorphism invariance, which has already been discussed in the context of asymptotic safety [22].

Let us finally illustrate the above discussion in the familiar setting of the Einstein-Hilbert truncation [6] in $d=4$ which is defined by the ansatz

$$
\bar{\Gamma}_{k}\left[g_{\mu v}\right]=-\frac{1}{16 \pi G_{k}} \int \mathrm{d}^{4} x \sqrt{g}\left(R(g)-2 \Lambda_{k}\right)
$$

Inserting (2.3) with $\phi=\phi(x)$ we obtain

$$
\bar{\Gamma}_{k}\left[\phi^{2} \hat{g}_{\mu v}\right]=\frac{3}{4 \pi G(k)} \int \mathrm{d}^{4} x \sqrt{\hat{g}}\left[-\frac{1}{2} \hat{g}^{\mu v} \partial_{\mu} \phi \partial_{\nu} \phi-\phi^{2}+\frac{1}{6} \Lambda(k) \phi^{4}\right]
$$

For $x$-independent $\phi$ only the potential term survives, with

$$
\begin{aligned}
U_{k}(\phi) & =\frac{3}{4 \pi G(k)}\left(-\phi^{2}+\frac{1}{6} \Lambda(k) \phi^{4}\right) \\
& =\frac{3}{4 \pi g(k)}\left(-k^{2} \phi^{2}+\frac{1}{6} \lambda(k) k^{4} \phi^{4}\right)
\end{aligned}
$$

If $\Lambda(k)>0$, the case we shall always consider in the following, $U_{k}(\phi)$ has a minimum at a nonzero radius given by

$$
\phi_{0}(k)=\sqrt{3 / \Lambda(k)}
$$


This is exactly the radius of the $S^{4}$ which solves the ordinary Einstein equation following from the action $(2.12)^{1}$. In the second line of (2.14) we employed the dimensionless Newton constant $g(k) \equiv$ $k^{2} G(k)$ and cosmological constant $\lambda(k) \equiv \Lambda(k) / k^{2}$. So there are the following two equivalent ways of writing the effective potential:

$$
\begin{aligned}
& U_{\mathrm{eff}}(\phi)=\frac{3}{4 \pi}\left[-\frac{1}{G\left(\phi^{-1}\right)} \phi^{2}+\frac{1}{6} \frac{\Lambda\left(\phi^{-1}\right)}{G\left(\phi^{-1}\right)} \phi^{4}\right] \\
& U_{\mathrm{eff}}(\phi)=\frac{3}{4 \pi}\left[-\frac{1}{g\left(\phi^{-1}\right)}+\frac{1}{6} \frac{\lambda\left(\phi^{-1}\right)}{g\left(\phi^{-1}\right)}\right]
\end{aligned}
$$

The RG trajectories of the Einstein-Hilbert truncation have been investigated and classified in [9]. Here we can concentrate on those with a positive cosmological constant, those of "Type IIIa". Important regimes along a Type IIIa trajectory include

The NGFP regime: $g(k) \approx g_{*}, \lambda(k) \approx \lambda_{*}$ for $k \gtrsim M$.

The $k^{4}$ regime: $G(k) \approx$ const, $\Lambda(k) \propto k^{4}$ for $k_{\mathrm{T}} \lesssim k \lesssim M$, where $k_{\mathrm{T}}$ is the "turning point" scale at which $\beta_{\lambda}$ vanishes.

The classical regime: $G(k) \approx$ const $\equiv \bar{G}, \Lambda(k) \approx$ const $\equiv \bar{\Lambda}$ for $k_{\text {term }} \ll k \lesssim k_{\mathrm{T}}$ where $k_{\text {term }}$ is the scale at which the Einstein-Hilbert truncation breaks down and the trajectory terminates at a singularity $^{2}$.

If one defines the classical Planck mass and length by $m_{\mathrm{Pl}} \equiv \ell_{\mathrm{Pl}}^{-1} \equiv \bar{G}^{-1 / 2}$ one finds that, approximately, $M \approx m_{\mathrm{Pl}}$. (For further details see [9,41,45]; see in particular Fig. 4 of [45].)

In the $k^{4}$-regime, when $k$ decreases, the cosmological constant quickly becomes smaller proportional to $k^{4}$, and the radius of the sphere "on shell", $\phi_{0}(k)$, increases proportional to $1 / k^{2}$.

If the underlying RG trajectory of QEG is of Type IIIa then $U_{\text {eff }}(\phi)$ is constant in the NGFP regime $\phi \lesssim \ell_{\mathrm{Pl}}$, and it equals the classical potential for $k_{\mathrm{T}}^{-1} \lesssim \phi \lesssim k_{\text {term. }}^{-1}$. Note that our ignorance about the infrared end of the trajectory entails that we have no information about the effective potential for large values of $\phi$. The intermediate $k^{4}$-regime of the trajectory gives rise to a behavior

$$
U_{\text {eff }}(\phi) \propto\left(-\phi^{2}+\text { const }\right) \text { for } k_{\mathrm{T}}^{-1} \lesssim \phi \lesssim \ell_{\mathrm{Pl}} .
$$

In the above discussion we tacitly assumed that the trajectory is such that $M^{2} \approx m_{\mathrm{Pl}}^{2} \gg \bar{\Lambda}$; otherwise no classical regime would exist.

A qualitative sketch of the resulting $U_{\text {eff }}$ is shown in Fig. 1. It is compared there to the classical potential $U_{\text {class }}$ which would obtain if $G$ and $\Lambda$ had no $k$-dependence at all. The crucial difference between the two is the almost constant $U_{\text {eff }}$ at small $\phi$. This regime is a pure quantum gravity effect, directly related to the existence of a NGFP. Quantum mechanically, but not classically, the universe can be stationary at small values of $\phi$, at least at $\phi=0$.

As a consequence of our assumption $\bar{\Lambda} \ll m_{\mathrm{Pl}}^{2}$, the $U_{\text {eff }}=$ const regime ends at a radius $\phi \approx \ell_{\mathrm{Pl}}$ which is smaller than the classical "on-shell" radius $\bar{\phi}_{0}=\sqrt{3 / \bar{\Lambda}}$. The actual "size of the universe" corresponds to a scale in the classical regime of the RG trajectory therefore.

\footnotetext{
${ }^{1}$ Because this space is maximally symmetric, by Palais' theorem [35], inserting the ansatz $g_{\mu \nu}=\phi^{2} \hat{g}_{\mu v}$ commutes with deriving the critical point.

${ }^{2}$ If one tentatively matches the trajectory against the observed values of $G$ and $\Lambda$ one finds that $k_{\mathrm{T}} \approx 10^{-30} \mathrm{~m}_{\mathrm{Pl}}$, corresponding to $k_{\mathrm{T}}^{-1} \approx 10^{-3} \mathrm{~cm}$, and $k_{\mathrm{term}} \approx 10^{-60} m_{\mathrm{Pl}} \approx H_{0}$ so that $k_{\mathrm{term}}^{-1}$ equals about the present Hubble radius [45], [41].
} 


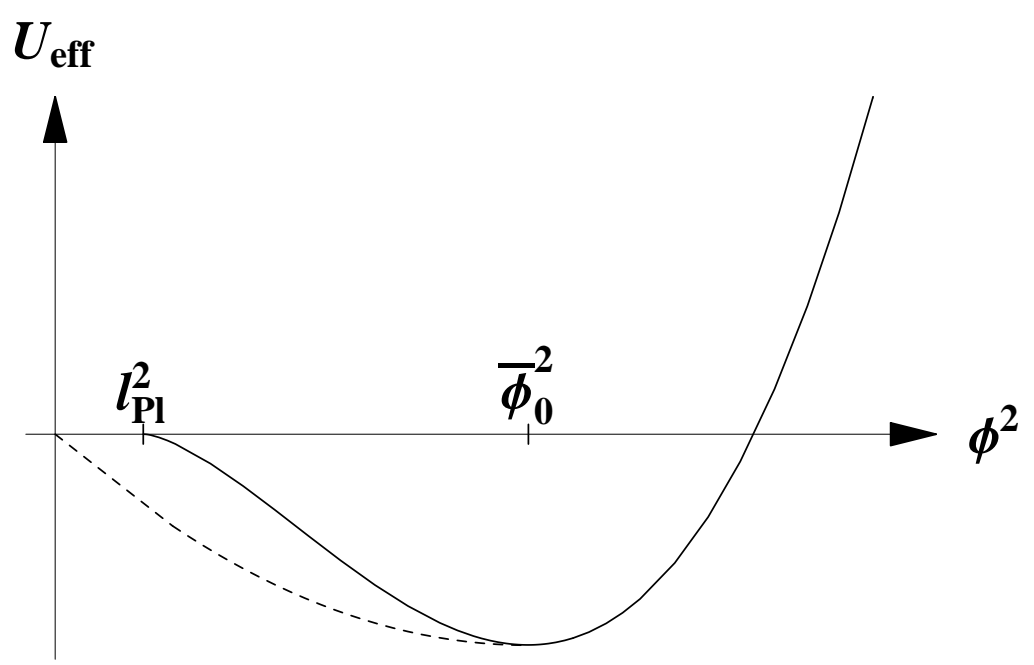

Figure 1: The effective potential for the Type IIIa trajectory discussed in the text. The dashed line represents the potential $U_{\text {class }}$ with the same values of $\bar{G}$ and $\bar{\Lambda}$, but all quantum effects neglected.

In the region where the quantum effects modify $U_{\text {class }}$ most strongly the term $\propto \phi^{2}$ is the dominant one. We can therefore say that the key effect behind the flattening of the potential near the origin is the running of Newton's constant. Its consequence for the shape of $U_{\text {eff }}$ can be understood as the result of the "RG improvement" [36-47]

$$
\frac{1}{G} \phi^{2} \longrightarrow \frac{1}{G\left(k=\phi^{-1}\right)} \phi^{2}=\frac{1}{g_{*}}
$$

with $G(k)=g_{*} / k^{2}$, as appropriate near the NGFP.

Up to now we considered pure gravity. However, including matter, the above argument will go through unaltered provided the matter contributions to the beta-functions do not destroy the NGFP. A detailled analysis showed [17] that the NGFP indeed persists for a wide class of matter systems. In these cases we would expect the same flattening of $U_{\text {eff }}(\phi)$ as for pure gravity.

\section{Possible Connections to Numerical Simulations within the CDT Approach}

The causal dynamical triangulation approach [48-51] defines a discrete version of the Wick rotated quantum-gravitational proper-time propagator

$$
G_{\Lambda, G}^{\mathrm{E}}\left[g_{3}(0), g_{3}(t)\right]=\int \mathscr{D} g_{\mathrm{E}} \mathrm{e}^{-S_{\mathrm{E}}\left[g_{\mathrm{E}}\right]}
$$

Here $S_{\mathrm{E}}$ is the Euclidean Einstein-Hilbert action, and the integration is over all 4-dimensional Euclidean geometries $g_{\mathrm{E}}$ of topology $S^{3} \times[0,1]$, each with proper-time running from 0 to $t$, and with prescribed spatial boundary geometries $g_{3}(0)$ and $g_{3}(t)$, respectively. In the numerical evaluation of (3.1), for technical reasons, periodic rather than fixed boundary conditions have been used so that the topology of the spacetimes summed over is $S^{3} \times S^{1}$ rather than $S^{3} \times[0,1]$. (Furthermore, 
the Monte-Carlo simulations typically are done at constant 4-volume $V_{4}$ rather than constant $\Lambda$; the corresponding propagator is related to (3.1) by a Laplace transformation.)

Remarkably, a nontrivial point of contact between CDT and QEG has been found already [23]: They both agree on the microscopic spectral dimension of macroscopically 4-dimensional spacetimes; in either case one finds the somewhat surprising result $d_{\mathrm{S}}=2[8,23]$. It is therefore tempting to ask whether the characteristic behavior of the conformal factor that we have discussed in the previous section might also be observed in the corresponding Monte-Carlo data provided by CDT.

First of all, it is instructive to visualize the typical, statistically representative 4-geometries contributing to the path integral. They are characterized by a function $V_{3}(s), 0 \leq s \leq t$, where $V_{3}(s)$ is the 3-volume of the spatial $S^{3}$ at proper-time $s$. If $t$ is large enough, a "typical universe" has long epochs with a very small $V_{3}$ at early and late times (the "stalk") and in between a region with a large $V_{3}(s)$, see Fig. 1 of ref. [49].

It has been shown [49] that the dynamics of these "universes" is well reproduced by a minisuperspace effective action for Wick rotated Robertson-Walker metrics

$$
\mathrm{d} s^{2}=\mathrm{d} t^{2}+a^{2}(t) \mathrm{d} \Omega_{3}^{2}
$$

where $\mathrm{d} \Omega_{3}^{2}$ is the line element of the unit 3-sphere so that $V_{3}(s) \propto a^{3}(s)$. It reads

$$
S_{\text {eff }}[a]=-\frac{3 \sigma_{3}}{8 \pi} \frac{1}{G} \int_{0}^{t} \mathrm{~d} s\left\{-a(s)\left(\frac{\mathrm{d} a(s)}{\mathrm{d} s}\right)^{2}+V(a(s))\right\}
$$

Classically, the potential $V$ is

$$
V(a)=-a+\frac{1}{3} \Lambda a^{3} \equiv V_{\mathrm{cl}}(a)
$$

The action (3.3) with (3.4) is, up to an overall minus sign, what one obtains when one inserts (3.2) into the Einstein-Hilbert action. (In simulations with fixed $V_{4}$ the constant $\Lambda$ is a Lagrange multiplier to be fixed such that $\int_{0}^{t} \mathrm{~d} s V_{3}(s)=V_{4}$.)

The challenge is now to determine numerically the effective action $S_{\text {eff }}[a]$ for small $a$ where we expect to see quantum corrections to the classical potential (3.4). Since the flattening of the effective potential occurs at conformal factors of the order of the Planck length and below, the corresponding lattice simulations require a lattice spacing whose physical size is of the same order of magnitude. Future simulations should be able to probe this regime. The prediction would then be a flattening $V(a) \approx$ const at small $a$.

In order to be able to confront possible future Monte-Carlo data with the prediction of QEG, two comments are appropriate. Firstly, upon introducing the conformal time $\eta(t)=\int^{t} \mathrm{~d} t^{\prime} / a\left(t^{\prime}\right)$ the line element (3.2) assumes a form analogous to (2.3),

$$
\mathrm{d} s^{2}=\phi(\eta)^{2}\left[\mathrm{~d} \eta^{2}+\mathrm{d} \Omega_{3}^{2}\right]
$$

with the conformal factor $\phi(\eta) \equiv a(t(\eta))$. Since $\phi$ and $a$ differ only by a time reparametrization, which is irrelevant here, the potentials $U_{\text {eff }}(\phi)$ and $V(a)$ are almost the same object. In particular we defined $U_{\text {eff }}(\phi)$ in terms of a functional integral (or the corresponding flow equation) which does not include the conformal zero mode, i. e. fluctuations which merely change the radius of the $S^{4}$. Likewise its CDT counterpart $V(a)$ results from integrating out all modes other than the 
spatially constant global scale. Furthermore, another minor difference between the QEG and CDT setting, respectively, is that $\hat{g}_{\mu v}$ is a metric on $S^{4}$, while $\mathrm{d} \eta^{2}+\mathrm{d} \Omega_{3}^{2}$ refers to $S^{3} \times[0,1]$ or $S^{3} \times S^{1}$. However, we do not expect such global issues to cause qualitative changes for small conformal factors.

\section{Summary}

We analyzed the effective potential of the conformal factor both in Quantum Einstein Gravity. We demonstrated that if QEG is asymptotically safe then it gives rise to a potential which becomes flat for $\phi \rightarrow 0$, allowing for a phase of gravity with vanishing metric expectation value. The argument assumes the existence of an underlying UV fixed point, but is exact otherwise. Since the effective potential is also accessible to numerical simulations, its "measurement" by means of Monte-Carlo techniques might provide further insights into the relation between QEG and the lattice approaches to quantum gravity [48-52].

Acknowledgement: We would like to thank J. Ambjørn, H. Hamber, R. Loll, and R. Williams for helpful discussions. 


\section{References}

[1] For general introductions see C. Kiefer, Quantum Gravity, Second Edition, Oxford Science Publications, Oxford (2007);

H.W. Hamber, Quantum Gravitation, Springer, Berlin (2009).

[2] A. Ashtekar, Lectures on non-perturbative canonical gravity, World Scientific, Singapore (1991);

A. Ashtekar and J. Lewandowski, Class. Quant. Grav. 21 (2004) R53.

[3] C. Rovelli, Quantum Gravity, Cambridge University Press, Cambridge (2004).

[4] Th. Thiemann, Modern Canonical Quantum General Relativity, Cambridge University Press, Cambridge (2007).

[5] S. Weinberg in General Relativity, an Einstein Centenary Survey, S.W. Hawking and W. Israel (Eds.), Cambridge University Press (1979);

S. Weinberg, hep-th/9702027 and preprint arXiv:0903.0568 [hep-th].

[6] M. Reuter, Phys. Rev. D 57 (1998) 971 and hep-th/9605030.

[7] D. Dou and R. Percacci, Class. Quant. Grav. 15 (1998) 3449.

[8] O. Lauscher and M. Reuter, Phys. Rev. D 65 (2002) 025013 and hep-th/0108040.

[9] M. Reuter and F. Saueressig, Phys. Rev. D 65 (2002) 065016 and hep-th/0110054.

[10] O. Lauscher and M. Reuter, Phys. Rev. D 66 (2002) 025026 and hep-th/0205062.

[11] O. Lauscher and M. Reuter, Class. Quant. Grav. 19 (2002) 483 and hep-th/0110021.

[12] O. Lauscher and M. Reuter, Int. J. Mod. Phys. A 17 (2002) 993 and hep-th/0112089.

[13] W. Souma, Prog. Theor. Phys. 102 (1999) 181.

[14] M. Reuter and F. Saueressig, Phys. Rev. D 66 (2002) 125001 and hep-th/0206145; Fortschr. Phys. 52 (2004) 650 and hep-th/0311056.

[15] A. Bonanno and M. Reuter, JHEP 02 (2005) 035 and hep-th/0410191.

[16] For reviews on QEG see: M. Reuter and F. Saueressig, arXiv:0708.1317 [hep-th]; O. Lauscher and M. Reuter in Quantum Gravity, B. Fauser,

J. Tolksdorf and E. Zeidler (Eds.), Birkhäuser, Basel (2007) and hep-th/0511260;

O. Lauscher and M. Reuter in Approaches to Fundamental Physics, I.-O. Stamatescu and E. Seiler (Eds.), Springer, Berlin (2007).

[17] R. Percacci and D. Perini, Phys. Rev. D 67 (2003) 081503; Phys. Rev. D 68 (2003) 044018; Class. Quant. Grav. 21 (2004) 5035.

[18] A. Codello and R. Percacci, Phys. Rev. Lett. 97 (2006) 221301;

A. Codello, R. Percacci and C. Rahmede, Int. J. Mod. Phys. A23 (2008) 143; preprint arXiv:0805.2909 [hep-th].

[19] D. Litim, Phys. Rev. Lett. 92 (2004) 201301; AIP Conf. Proc. 841 (2006) 322;

P. Fischer and D. Litim, Phys. Lett. B 638 (2006) 497;

AIP Conf. Proc. 861 (2006) 336.

[20] P. Machado and F. Saueressig, Phys. Rev. D 77 (2008) 124045;

D. Benedetti, P. Machado and F. Saueressig, preprint arXiv:0901.2984 [hep-th]. 
[21] M. Reuter and H. Weyer, Phys. Rev. D 79ă(2009) 105005 and arXiv:0801.3287 [hep-th].

[22] M. Reuter and H. Weyer, Phys. Rev. D, in press and preprint arXiv:0804.1475 [hep-th].

[23] O. Lauscher and M. Reuter, JHEP 10 (2005) 050 and hep-th/0508202.

[24] M. Reuter and J.-M. Schwindt, JHEP 01 (2006) 070 and hep-th/0511021.

[25] M. Reuter and J.-M. Schwindt, JHEP 01 (2007) 049 and hep-th/0611294.

[26] F. Neugebohrn, arXiv:0704.3205 [hep-th].

[27] P. Forgács and M. Niedermaier, hep-th/0207028;

M. Niedermaier, JHEP 12 (2002) 066; Nucl. Phys. B 673 (2003) 131;

Class. Quant. Grav. 24 (2007) R171.

[28] For detailed reviews of asymptotic safety in gravity see:

M. Niedermaier and M. Reuter, Living Reviews in Relativity 9 (2006) 5;

R. Percacci, arXiv:0709.3851 [hep-th].

[29] J.-E. Daum and M. Reuter, Adv. Sci. Lett. 2 (2009) 255 and arXiv:0806.3907 [hep-th].

[30] C. Wetterich, Phys. Lett. B 301 (1993) 90.

[31] M. Reuter and C. Wetterich, Nucl. Phys. B 417 (1994) 181, Nucl. Phys. B 427 (1994) 291,

Nucl. Phys. B 391 (1993) 147, Nucl. Phys. B 408 (1993) 91;

M. Reuter, Phys. Rev. D 53 (1996) 4430, Mod. Phys. Lett. A 12 (1997) 2777.

[32] J. Berges, N. Tetradis and C. Wetterich, Phys. Rep. 363 (2002) 223;

C. Wetterich, Int. J. Mod. Phys. A 16 (2001) 1951.

[33] For reviews of the effective average action in Yang-Mills theory see:

M. Reuter, hep-th/9602012; J. Pawlowski, hep-th/0512261; H. Gies, hep-ph/0611146.

[34] H.W. Hamber, Phys. Rev. D 45 (1992) 507;

Phys. Rev. D 61 (2000) 124008; arXiv:0704.2895 [hep-th];

T. Regge and R.M. Williams, J. Math. Phys. 41 (2000) 3964.

[35] R.S. Palais, Comm. Math. Phys. 69 (1997) 19.

[36] A. Bonanno and M. Reuter, Phys. Rev. D 62 (2000) 043008 and hep-th/0002196; Phys. Rev. D 73 (2006) 083005 and hep-th/0602159;

Phys. Rev. D 60 (1999) 084011 and gr-qc/9811026.

[37] M. Reuter and E. Tuiran in Proceedings of the Eleventh Marcel Grossmann Meeting on General Relativity,

H. Kleinert, R.T. Jantzen and R. Ruffini (Eds.), World Scientific, Singspore (2008) and hep-th/0612037.

[38] A. Bonanno and M. Reuter, Phys. Rev. D 65 (2002) 043508 and hep-th/0106133.

[39] M. Reuter and F. Saueressig, JCAP 09 (2005) 012 and hep-th/0507167.

[40] A. Bonanno and M. Reuter, Phys. Lett. B 527 (2002) 9 and astro-ph/0106468;

Int. J. Mod. Phys. D 13 (2004) 107 and astro-ph/0210472;

E. Bentivegna, A. Bonanno and M. Reuter,

JCAP 01 (2004) 001 and astro-ph/0303150.

[41] A. Bonanno and M. Reuter, JCAP 08 (2007) 024 and arXiv:0706.0174 [hep-th]. 
[42] A. Bonanno, G. Esposito and C. Rubano, Gen. Rel. Grav. 35 (2003) 1899; Class. Quant. Grav. 21 (2004) 5005;

A. Bonanno, G. Esposito, C. Rubano and P. Scudellaro, Class. Quant. Grav. 23 (2006) 3103 and 2 (2007) 1443.

[43] M. Reuter and H. Weyer, Phys. Rev. D 69 (2004) 104022 and hep-th/0311196.

[44] M. Reuter and H. Weyer, Phys. Rev. D 70 (2004) 124028 and hep-th/0410117.

[45] M. Reuter and H. Weyer, JCAP 12 (2004) 001 and hep-th/0410119.

[46] F. Girelli, S. Liberati, R. Percacci and C. Rahmede, Class. Quant. Grav. 24 (2007) 3995.

[47] J. Moffat, JCAP 05 (2005) 2003;

J.R. Brownstein and J. Moffat, Astrophys. J. 636 (2006) 721;

Mon. Not. Roy. Astron. Soc. 367 (2006) 527.

[48] J. Ambjørn, J. Jurkiewicz and R. Loll, Phys. Rev. Lett. 93 (2004) 131301.

[49] J. Ambjørn, J. Jurkiewicz and R. Loll, Phys. Lett. B 607 (2005) 205.

[50] J. Ambjørn, J. Jurkiewicz and R. Loll, Phys. Rev. Lett. 95 (2005) 171301; Phys. Rev. D 72 (2005) 064014; Contemp. Phys. 47 (2006) 103.

[51] J. Ambjørn, A. Görlich, J. Jurkiewicz and R. Loll, Phys. Rev. Lett. 100 (2008) 091304.

[52] H.W. Hamber, arXiv: 0704.2895 [hep-th]; Gen. Rel. Grav. 41 (2009) 817. 